\title{
Equinococosis/hidatidosis en la VII Región de Chile: diagnóstico e intervención educativa
}

\author{
Werner Apt, ${ }^{1}$ Carlos Pérez, ${ }^{1}$ Ercira Galdamez, ${ }^{2}$ Sergio Campano, ${ }^{3}$ \\ Flavio Vega, ${ }^{4}$ Danilo Vargas, ${ }^{1}$ Jorge Rodríguez, ${ }^{5}$ Clara Retamal, ${ }^{1}$ \\ Pedro Cortés, ${ }^{6}$ Inés Zulantay y Paul H. de Rycke ${ }^{7}$
}

RESUMEN El presente estudio se proyectó para un trabajo simultáneo en tres ámbitos: a) diagnóstico serológico y radiológico y tratamiento quirúrgico de la hidatidosis en la población humana asintomática; b) diagnóstico animal y tratamiento de los perros, y c) evaluación de conocimientos e intervención educativa en familias campesinas y en profesionales y técnicos en salud, agropecuaria y educación, con el fin de contribuir al control del ciclo de transmisión de la enfermedad. Se efectuaron pruebas de hemaglutinación indirecta y ELISA a 5566 personas aparentemente sanas. Los $42(0,8 \%)$ casos con resultados positivos en ambas pruebas (seroprevalencia de 754,6 por 100 000) fueron citados para ser sometidos a una ecografía hepática y una radiografía de tórax y, de los 26 que acudieron, 16 presentaron imágenes compatibles con quiste hidatídico. Estos 16 casos fueron enviados al hospital para ser intervenidos y en los 9 que acudieron se confirmó el diagnóstico quirúrgicamente, lo cual representa una prevalencia de 161,7 por 100000. En 2358 perros se procedió a la detección de la forma estrobilar de Echinococcus granulosus mediante purga con bromhidrato de arecolina y se obtuvieron resultados positivos en $11 \%$. Los datos oficiales registrados en los mataderos revelaron la presencia de quistes hidatídicos en $13 \%$ de los bovinos, $4,4 \%$ de los ovinos y 4,2\% de los porcinos sacrificados en la región. El programa educativo comprendió una evaluación de conocimientos mediante una encuesta al cabeza de familia, la intervención educativa entre las familias por un proceso participatorio activo no formal de carácter lúdico en el que participaron 1082 familias, y la intervención educativa entre profesionales y técnicos mediante metodología a distancia y presencial. Para evaluar los resultados del programa se compararon los resultados de las pruebas de conocimientos antes y después de la intervención educativa en 200 familias que participaron en ella (casos) y en 95 que no participaron (controles). Del análisis de los conocimientos sobre equinococosis/hidatidosis, 783 familias (55\%) demostraron no saber nada sobre la infección. Se observó que las técnicas participatorias lúdicas se adaptan a la forma de ser del campesino y permiten obtener cambios. Se capacitaron 276 profesionales de la salud, 201 auxiliares técnicos y 453 profesores rurales. Entre los equipos de atención primaria rural, el programa tuvo una cobertura de $100 \%$.

1 Instituto de Ciencias Biomédicas, Programa de Parasitología, Facultad de Medicina. Universidad de Chile, Santiago. Enviar toda la correspondencia a Werner Apt a la siguiente dirección postal: Casilla 427, Santiago 3, Chile. Fax (56-2) 7356579. Teléfonos (56-2) 6786122/ 6786114 .

2 Escuela de Enfermería, Facultad de Medicina, Universidad de Chile, Santiago, Chile.
3 Servicio Agrícola y Ganadero, Ministerio de Agricultura, Santiago, Chile.

4 Médico Veterinario. VII Región, Chile.

5 Escuela de Salud Pública, Facultad de Medicina, Universidad de Chile, Santiago, Chile.

6 Hospital del Pino, Servicio de Salud Metropolitano, Santiago, Chile.

7 Laboratorio de Zoofisiología, Facultad Wetenschappen, Universidad de Gante, Gante, Bélgica.
La hidatidosis es una zoonosis parasitaria propia de las regiones agrícolas y ganaderas. En América Latina se presenta con mayor frecuencia en Argentina, Brasil (Río Grande do Sul), Chile, Perú y Uruguay (1). En Chile, la frecuencia acumulada de la hidatido- 
sis humana entre 1961 y 1992 fue de 19119 casos, con 1625 muertes que representan una tasa de letalidad de $8,5 \%$. Las tasas medias anuales de incidencia y prevalencia son de 4,4 y 4,5 por 100000 habitantes, respectivamente, con variaciones regionales. Datos oficiales correspondientes al trienio de 1986 a 1988 señalaban que, en Chile, $16 \%$ de los casos pertenecían a la XI Región, $11 \%$ a la VII Región y 7\% a la XII Región (2). Las tasas medias de incidencia y prevalencia acumulada calculadas en ese período fueron de 7,1 y 6,3 por 100000 habitantes para todo el país, 12,9 y 11,6 para la VII Región, 189,7 y 183,2 para la XI Región y 42,6 y 39,9 para la XII Región. Las cifras registradas en la VII Región fueron inferiores a las de la XI y la XII, a pesar de que en estas últimas el Ministerio de Agricultura, a través del Servicio Agrícola y Ganadero (SAG), había implementado programas de control pecuario en 1979 y 1981, respectivamente $(3,4)$. Los programas del SAG se basan en el bloqueo del ciclo de transmisión mediante el tratamiento periódico del huésped definitivo (el perro) con prazicuantel y medidas de saneamiento básico para evitar riesgos epidémicos. Este servicio, por depender del Ministerio de Agricultura, orienta su quehacer preferentemente en el aspecto agropecuario.

La VII Región, que contaba en 1992 con 836141 habitantes y una ruralidad de $40 \%$, está formada por cuatro provincias que, de Sur a Norte, son: Cauquenes, Linares, Talca y Curicó. En el período de 1986 a 1988, las tasas de incidencia por 100000 habitantes superaban a la nacional en todas las provincias (108 en Cauquenes, 8,8 en Linares y 7,4 en Talca), excepto en Curicó $(0,47)$.

En Chile, entre 1983 y 1988, las pérdidas por decomiso de hígados y riñones habrían tenido un valor aproximado de 6,4 millones de dólares estadounidenses (US\$), es decir, más de un millón al año (2). En la población humana, aunque hay muchos otros factores que se pueden cuantificar (5), considerando solo los costos de hospitalización, cuya duración media es de 22 días, el gasto ascendía en 1992 a
US\$ 2000 por paciente, pero la cifra es todavía mayor si se producen complicaciones de la cirugía hepática o pulmonar (las intervenciones más frecuentes) o si se trata de una hidatidosis ósea, cardíaca o encefálica.

Los datos expuestos pueden explicarse por la frecuente presencia del perro y la actividad ganadera, de manera que el encuentro ganadoperro-ganado o ganado-perro-hombre se produce de forma natural, coadyuvado por la ruralidad y por las condiciones favorables al ciclo de transmisión que produce el habitante rural al permitir que los perros domésticos se alimenten con vísceras crudas de animales infectados $(6,7)$. Como los datos disponibles provenían de pacientes que consultaban y no se conocía la proporción de reservorios infectados, se inició esta investigación con un equipo multidisciplinario encabezado por la Unidad de Parasitología de la Universidad de Chile y con la participación de los Ministerios de Salud y Educación.

Los objetivos consistieron en determinar la prevalencia de la infección en la población general asintomática y en los perros y en evaluar los conocimientos, prácticas y creencias de la población sobre el problema. Se programó una intervención dirigida al diagnóstico, prevención y control de la infección en las cuatro provincias de la VII Región. Para ello, se dotó a los laboratorios de los hospitales provinciales con nuevas técnicas diagnósticas, se capacitó a parte de su personal, se desparasitaron los perros estudiados y se estableció una red educativa cuyos puntos clave fueron las familias, las escuelas y los servicios de salud de atención primaria y hospitalaria.

\section{MATERIALES Y MÉTODOS}

El estudio, realizado por un equipo multidisciplinario entre 1992 y 1997, se proyectó para un trabajo simultáneo en tres ámbitos: a) diagnóstico serológico y radiológico y tratamiento quirúrgico de la población humana asintomática; b) diagnóstico animal y tratamiento de los perros, y c) evalua- ción de conocimientos e intervención educativa en familias campesinas. El programa educativo se hizo extensivo a profesionales y técnicos en salud, agropecuaria y educación.

Se trabajó con una muestra poblacional seleccionada probabilísticamente del área rural de la VII Región. Como unidad de muestreo se utilizó la vivienda. El tamaño mínimo de la muestra se calculó considerando una tasa de seroprevalencia de 600 por 100000 en la población presuntamente sana, un nivel de confianza de $95 \%$ y una precisión de $0,5 \%$. Aplicando la fórmula $n=\mathrm{Z}_{0,025}^{2} \cdot \mathrm{P} \cdot \mathrm{Q} / \mathrm{E}^{2}$, donde $\mathrm{Z}=1,96, \mathrm{P}=600, \mathrm{Q}=99400$ y $\mathrm{E}=500$, se calculó un tamaño muestral mínimo de 917 viviendas. En total se estudiaron 1423 viviendas, distribuidas proporcionalmente a la población de cada provincia: 424 en Cauquenes, 268 en Curicó, 439 en Linares y 292 en Talca.

Las unidades de observación para el diagnóstico de la hidatidosis estuvieron constituidas por todos los miembros de la familia y, al aplicar el índice de personas por familia, que al inicio de la investigación era de 4,5, se calculó un número aproximado de 6400 participantes. Para el diagnóstico de la equinococosis, las unidades de observación fueron todos los perros presentes en cada casa, exceptuando los cachorros y hembras preñadas, en los que está contraindicada la administración del tenífugo utilizado (bromhidrato de arecolina).

El estudio serológico se efectuó solo en personas que aceptaron el procedimiento y dieron su consentimiento tras recibir la información pertinente. Se excluyó a los menores de 3 años. A cada persona se le extrajeron $10 \mathrm{~mL}$ de sangre, a los que no se añadió anticoagulante. Los sueros se sometieron primero a la reacción de "dot"-ELISA para investigar su utilidad como prueba de tamizaje. Como pruebas diagnósticas se utilizaron la hemaglutinación indirecta (HAI) y la prueba de ELISA. Inicialmente, en los casos dudosos se recurrió a la doble difusión para la detección de anticuerpos frente al antígeno 5 (DD5) como prueba de confirmación. A las personas con resultados positivos en las pruebas de 
HAI y ELISA se les registró en una ficha clínica ad hoc para efectuarles una radiografía de tórax y una ecografía abdominal. A aquellas con serología y pruebas de imagen compatibles con la presencia de quiste hidatídico se les derivó al hospital provincial o regional para ser intervenidas quirúrgicamente.

La reacción de HAI, realizada con la técnica de Knierim y Saavedra (8), se consideró positiva cuando los títulos fueron $\geq 1 / 100$; la prueba de ELISA, efectuada con la técnica descrita por Vargas et al. (9), se consideró positiva cuando los resultados fueron superiores al valor de corte en tres o más desviaciones estándar; la DD5, realizada con la técnica de Coltorti y VarelaDíaz (10), se consideró positiva en presencia del arco 5 de Caprón o de dos o más bandas nítidas, y la prueba "dot"ELISA, practicada con la técnica de Vargas et al. (11), se consideró positiva cuando la intensidad de la señal de la muestra problema fue superior a la obtenida con los controles negativos. Los equipos locales fueron capacitados para realizar esta última prueba, que se efectuó en los laboratorios de los hospitales provinciales y regional; sus resultados fueron confirmados en el laboratorio de inmunodiagnóstico de la Facultad de Medicina, Sur, en el cual se llevaron a cabo todas las pruebas de HAI, ELISA y DD5.

Para el diagnóstico de la equinococosis, basado en la demostración de la presencia de la forma estrobilar de Echinococcus granulosus, se empleó la purga con el tenífugo bromhidrato de arecolina $(5 \mathrm{mg} / \mathrm{Kg})$, descrita por Schantz (12). El diagnóstico solo se efectuó en animales que evacuaron la fracción mucosa, que fue incluida en bandejas de fondo oscuro con auxilio de una lupa manual. El hallazgo de un solo ejemplar de E. granulosus fue suficiente para considerar positivo al perro. Se mantuvieron rigurosas medidas de seguridad para evitar tanto las infecciones de los operadores y personas asistentes como la contaminación del medio ambiente. Los perros positivos se trataron con prazicuantel $(5 \mathrm{mg} / \mathrm{Kg}$ ).

La evaluación de los conocimientos de las familias se realizó a través de una entrevista al cabeza de familia en la que se recogió información acerca de los antecedentes familiares generales y de los antecedentes personales de sus miembros, así como de los conocimientos, hábitos y conductas relacionados con la equinococosis/hidatidosis.

La intervención educativa familiar se programó para al menos un integrante de cada hogar encuestado y se efectuó por la vía no formal. Por lo complejo del tema, el proceso estuvo mediado por educadoras entrenadas en metodologías de educación de adultos y con formación específica sobre la hidatidosis/equinococosis. Se diseñaron tres juegos. El primero lo desarrollaron las familias después de la entrevista y su objetivo consistió en motivar la asistencia al proceso educativo; el segundo proporcionó información sobre el ciclo, mecanismo de transmisión, control y prevención de la parasitosis, y el tercero tuvo por objetivo evaluar el aprendizaje y lograr una suscripción de compromiso de las familias para la prevención y el control de la infección. El proceso educativo se evaluó mediante la realización de una prueba de conocimientos antes y tres meses después de la intervención. Esta evaluación se realizó en un grupo de 200 familias que participaron en el proceso educativo, definidas como casos, y en un grupo de otras 95 familias que no participaron en el mismo, definidas como controles. Los resultados de ambos grupos en las pruebas de conocimientos anterior y posterior a la intervención educativa fueron comparados con la prueba de Wilcoxon para datos apareados (antes y después de la intervención educativa) y la $t$ de Student para datos independientes (casos y controles). Posteriormente, los controles también fueron sometidos a la intervención educativa con la misma metodología que las demás familias.

La intervención educativa entre profesionales y técnicos fue abordada por la vía formal, para lo cual se crearon programas de capacitación organizados en conjunto con las entidades participantes: servicios provinciales de capacitación de hospital y de atención primaria, Instituto de Desarrollo Agropecuario, Centro de Perfeccionamiento e Investigaciones Pedagógicas del Mi- nisterio de Educación, Departamentos de Educación e Ilustres Municipalidades provinciales, y Centro de Extensión Biomédica de la Facultad de Medicina de la Universidad de Chile. Debido al gran número de educandos previstos por provincia y año de desarrollo del programa, se diseñó una metodología mixta a distancia, con material de autoinstrucción, y presencial, con reuniones evaluativas de carácter formativo y con fines calificativos. La evaluación de este proceso se efectuó mediante la comparación de una prueba de conocimientos antes y después de la intervención. En el caso particular de los profesores rurales se les exigió la elaboración de un proyecto de transferencia al aula por cada microcentro del Programa de Mejoramiento de la Calidad y Equidad de la Educación Rural que participó en el estudio.

\section{RESULTADOS}

El grupo de estudio quedó constituido por 1423 familias con un total de 6561 miembros (media de 4,6 personas por familia). De la totalidad de los individuos estudiados, $51,2 \%$ fueron mujeres y $48,8 \%$ hombres. La distribución por edades fue la siguiente: $30,9 \%$ de niños (0-14 años), 8,4\% de adolescentes (15 a 19 años) y 60,7\% de adultos (mayores de 20 años). El número medio de perros por familia en la región fue de 2,2: 2,7 en Talca, 2,6 en Linares, 0,7 en Curicó y 2,4 en Cauquenes.

Presentaron algún grado de escolaridad 5430 personas $(82,8 \%)$, de las cuales 4507 (83\%) habían obtenido algún nivel de enseñanza básica, 835 $(15,4 \%)$ algún curso de enseñanza media y $88(1,6 \%)$ algún curso técnico; otras $486(7,4 \%)$ todavía no habían ingresado en el sistema educativo y 645 $(9,8 \%)$ no habían asistido al colegio. De 6115 personas en edad de haber recibido algún grado de alfabetización, solo $4892(80 \%)$ estaban realmente alfabetizadas.

La distribución por ocupación fue la siguiente: amas de casa, 27,4\%; sector agrícola, ya sea en calidad de propietario, arrendatario, administrador, encargado o trabajador, 25,3\%, y estu- 
CUADRO 1. Resultados de las pruebas de ELISA y hemaglutinación indirecta (HAI) para hidatidosis en habitantes rurales de la VII Región, según provincias. Chile, 1992-1997

\begin{tabular}{|c|c|c|c|c|c|}
\hline \multirow[b]{3}{*}{ Provincia } & \multirow[b]{3}{*}{$n$} & \multicolumn{4}{|c|}{ Reacciones positivas } \\
\hline & & \multicolumn{2}{|c|}{ ELISA } & \multicolumn{2}{|c|}{$\mathrm{HAI}$} \\
\hline & & No. & $\%$ & No. & $\%$ \\
\hline Cauquenes & 1290 & 65 & 5,0 & 26 & 2,0 \\
\hline Curicó & 978 & 87 & 8,9 & 3 & 0,3 \\
\hline Linares & 1713 & 131 & 7,6 & 21 & 1,2 \\
\hline Talca & 1585 & 118 & 7,4 & 13 & 0,8 \\
\hline Total & 5566 & 401 & 7,2 & 63 & 1,1 \\
\hline
\end{tabular}

diantes, 25\%. El resto no tenía una ocupación específica.

En 5566 personas se efectuaron exámenes serológicos mediante HAI y ELISA, cuyos resultados se detallan en el cuadro 1. La prueba de ELISA proporcionó resultados positivos en $66,7 \%$ de los individuos con HAI positiva y resultados negativos en 93,5\% de los individuos con HAI negativa. A su vez, la HAI solo identificó como positivos a $10,5 \%$ de los casos con prueba de ELISA positiva, pero coincidió en señalar como negativos a 99,6\% de los casos con prueba de ELISA negativa. Se encontró una asociación estadísticamente significativa $\left(\chi^{2}=16,46 ; P<\right.$ 0,001 ) entre los resultados de ambas pruebas y su concordancia también fue estadísticamente significativa $(\kappa=$ $0,1647 ; P<0,001)$. Ambas pruebas fueron positivas en 42 individuos $(0,8 \%)$, que constituyeron el grupo de candidatos a la realización de pruebas de visualización. Todos estos casos fueron citados a los hospitales correspondientes por los encargados de los servicios locales a través de la red de postas rurales y las citas fueron refrendadas por el equipo de campo del proyecto. Acudieron 26 personas $(61,9 \%)$ a las que se efectuaron 52 exámenes (26 radiografías de tórax y 26 ecografías abdominales). Dieciséis de los 26 individuos $(61,5 \%)$ presentaron imágenes ecográficas $(10 / 26 ; 38,5 \%)$ o radiográficas $(6 / 26 ; 23 \%)$ compatibles con hidatidosis, diagnóstico que se confirmó quirúrgicamente en los nueve casos
$(56,3 \%)$ intervenidos; los otros siete no acudieron a la cita.

A 4577 personas se les efectuó la prueba de "dot"-ELISA, que fue positiva en 585 (12,8\%); dado su escaso rendimiento como prueba de tamizaje, en el último año del estudio se abandonó su realización.La DD5 se aplicó a 1977 individuos y fue positiva en 29 $(1,5 \%)$. Esta prueba se utilizó inicialmente en los casos dudosos por su alta especificidad, pero, posteriormente, al compararla con la HAI y la prueba de ELISA se observaron resultados concordantes; por este motivo y su alto costo, no se utilizó en todos los casos.

En el cuadro 2 se describe la frecuencia de la equinococosis en perros rurales de las diferentes provincias de la VII Región. De 2358 perros examinados, $1932(81,9 \%)$ reaccionaron al bromhidrato de arecolina $\mathrm{y}$, de estos, $11,0 \%$ padecían equinococosis. Los porcentajes más altos se obtuvieron en Cauquenes y Linares: 17,7 y 12,8\% respectivamente. El cuadro 3 muestra la frecuencia de hidatidosis en el ganado entre 1990 y 1995, según los datos oficiales de los mataderos provinciales.

De 1423 familias, 1051 (73,8\%) informaron que sacrificaban animales para consumo propio (cuadro 4) y, de las 1038 que lo hacían en casa, 924 $(64,9 \%)$ proporcionaron detalles sobre el sistema usado para desechar las vísceras (cuadro 5). De 786 familias que eliminaban las vísceras con riesgo de mantener el ciclo parasitario, 446 $(56,7 \%)$ las utilizaban para alimentar directamente a sus perros, y las otras $340(43,3 \%)$ las tiraban a la basura o al suelo en lugares alejados de la casa, con lo que podían llegar indirectamente al huésped definitivo.

De acuerdo con los conocimientos que manifiestaron los cabezas de familia acerca de la hidatidosis/equinococosis, 55\% de las familias (783/ 1423) declararon no saber nada $y$ $38,6 \%$ (549/1423) declararon saber algo, pero al examinar los conceptos emitidos, ya fuera sobre el ciclo de transmisión, los huéspedes por los que pasa el parásito en su desarrollo, el

CUADRO 2. Equinococosis en perros rurales de la VII Región, según provincia. Chile, 1992-1997

\begin{tabular}{|c|c|c|c|c|c|}
\hline \multirow[b]{2}{*}{ Provincia } & \multirow[b]{2}{*}{$n$} & \multicolumn{2}{|c|}{ Perros purgados ${ }^{\mathrm{a}}$} & \multicolumn{2}{|c|}{ Perros positivos } \\
\hline & & No. & $\%$ & No. & $\%$ \\
\hline Cauquenes & 649 & 575 & 88,6 & 102 & 17,7 \\
\hline Curicó & 381 & 291 & 76,4 & 11 & 3,8 \\
\hline Linares & 774 & 632 & 81,7 & 81 & 12,8 \\
\hline Talca & 554 & 434 & 78,3 & 19 & 4,4 \\
\hline Total & 2358 & 1932 & 81,9 & 213 & 11,0 \\
\hline
\end{tabular}

a La purga se realizó con bromhidrato de arecolina ( $5 \mathrm{mg} / \mathrm{Kg}$ de peso).

CUADRO 3. Hidatidosis en mataderos de la VII Región, según especie y provincia. Chile, 1990-1995

\begin{tabular}{|c|c|c|c|c|c|c|c|c|c|}
\hline \multirow[b]{3}{*}{ Provincia } & \multicolumn{3}{|c|}{ Bovinos } & \multicolumn{3}{|c|}{ Ovinos } & \multicolumn{3}{|c|}{ Porcinos } \\
\hline & \multirow[b]{2}{*}{ Sacrificados } & \multicolumn{2}{|c|}{ Infectados } & \multirow[b]{2}{*}{ Sacrificados } & \multicolumn{2}{|c|}{ Infectados } & \multirow[b]{2}{*}{ Sacrificados } & \multicolumn{2}{|c|}{ Infectados } \\
\hline & & No. & $\%$ & & No. & $\%$ & & No. & $\%$ \\
\hline Cauquenes & 16995 & 4623 & 27,2 & 3960 & 317 & 8,0 & 23151 & 2986 & 12,9 \\
\hline Curicó & 75787 & 12353 & 16,3 & 17553 & 632 & 3,6 & 166726 & 2501 & 1,5 \\
\hline Linares & 49039 & 6620 & 13,5 & 19745 & 1481 & 7,5 & 54547 & 4855 & 8,9 \\
\hline Talca & 103431 & 8171 & 7,9 & 27390 & 575 & 2,1 & 69415 & 2707 & 3,9 \\
\hline Total & 245252 & 31767 & 13,0 & 68648 & 3005 & 4,4 & 313839 & 13049 & 4,2 \\
\hline
\end{tabular}


CUADRO 4. Distribución de las familias según la provincia y la práctica utilizada en el sacrificio de animales para consumo propio. VII Región, Chile, 1992-1997

\begin{tabular}{|c|c|c|c|c|c|c|c|c|c|}
\hline \multirow[b]{3}{*}{ Provincia } & \multirow{3}{*}{$\begin{array}{l}\text { No. de } \\
\text { familias }\end{array}$} & \multicolumn{8}{|c|}{ Sacrificio de animales } \\
\hline & & \multicolumn{2}{|c|}{ En casa } & \multicolumn{2}{|c|}{$\begin{array}{c}\text { En } \\
\text { matadero }\end{array}$} & \multicolumn{2}{|c|}{$\begin{array}{c}\text { No } \\
\text { sacrifican }\end{array}$} & \multicolumn{2}{|c|}{$\begin{array}{c}\text { Dato } \\
\text { ausente }\end{array}$} \\
\hline & & No. & $\%$ & No. & $\%$ & No. & $\%$ & No. & $\%$ \\
\hline Cauquenes & 424 & 386 & 91,0 & 4 & 0,9 & 26 & 6,2 & 8 & 1,9 \\
\hline Curicó & 268 & 159 & 59,3 & 3 & 1,2 & 95 & 35,4 & 11 & 4,1 \\
\hline Linares & 439 & 324 & 73,8 & 3 & 0,7 & 105 & 23,9 & 7 & 1,6 \\
\hline Talca & 292 & 169 & 57,9 & 3 & 1,0 & 104 & 35,6 & 16 & 5,5 \\
\hline Total & 1423 & 1038 & 72,9 & 13 & 0,9 & 330 & 23,2 & 42 & 3,0 \\
\hline
\end{tabular}

CUADRO 5. Riesgo del sistema de eliminación de vísceras y destino de las mismas declarado por 924 familias según provincia. VII Región, Chile, 1992-1997

\begin{tabular}{|c|c|c|c|c|c|c|c|}
\hline \multirow[b]{3}{*}{ Provincia } & \multirow{3}{*}{$\begin{array}{l}\text { No. de } \\
\text { familias }\end{array}$} & \multicolumn{4}{|c|}{ Con riesgo } & & \\
\hline & & \multicolumn{2}{|c|}{$\begin{array}{l}\text { Las come } \\
\text { el perro }\end{array}$} & \multicolumn{2}{|c|}{$\begin{array}{c}\text { Van a la basura } \\
\text { o al suelo }\end{array}$} & \multicolumn{2}{|c|}{ Sin riesgo } \\
\hline & & No. & $\%$ & No. & $\%$ & No. & $\%$ \\
\hline Cauquenes & 383 & 164 & 42,8 & 176 & 46,0 & 43 & 11,2 \\
\hline Curicó & 131 & 69 & 52,7 & 49 & 37,4 & 13 & 9,9 \\
\hline Linares & 261 & 140 & 53,6 & 67 & 25,7 & 54 & 20,7 \\
\hline Talca & 149 & 73 & 49,0 & 48 & 32,2 & 28 & 18,8 \\
\hline Total & 924 & 446 & 48,3 & 340 & 36,8 & 138 & 14,9 \\
\hline
\end{tabular}

CUADRO 6. Población educada para el control de la hidatidosis perteneciente a las familias que voluntariamente asistieron al proceso educativo según provincia. VII Región, Chile, 1992-1997

\begin{tabular}{lcccc}
\hline \multirow{2}{*}{$\begin{array}{c}\text { Familias } \\
\text { Provincia }\end{array}$} & \multicolumn{2}{c}{ Familias educadas } & Personas \\
\cline { 3 - 4 } & encuestadas & No. & $\%$ & asistentes \\
\hline Cauquenes & 424 & 260 & 61,3 & 554 \\
Curicó & 268 & 232 & 82,9 & 559 \\
Linares & 439 & 353 & 80,4 & 595 \\
Talca & 292 & 237 & 81,2 & 519 \\
Total & 1423 & 1082 & 76,0 & $2227^{\mathrm{a}}$ \\
\hline
\end{tabular}

${ }^{a}$ De un total de 6115 personas con edad adecuada para participar en el programa educativo.

mecanismo de transmisión, su control o su prevención, solo se pudieron rescatar conceptos útiles para reforzar en 38 de ellas (7,0\%). En 91 de estas 549 familias $(16,6 \%)$ se evidenciaron conceptos errados que les daban una falsa seguridad que transmitían a su descendencia. Las 91 familias restantes $(6,4 \%)$ no se manifestaron de forma afirmativa ni negativa, sino que con- testaron la alternativa "otra" con opciones que no constituían situaciones de riesgo; no obstante, a la hora de ejecutar el proceso educativo fue necesario favorecer el desaprendizaje antes de plantear los conceptos correctos.

De las 1423 familias invitadas al encuentro con el equipo de educadores, se educaron $1082(76 \%)$, incluidas las 95 que sirvieron como controles. No obstante, muchas de las familias asistentes acudieron con algún otro miembro acompañando al cabeza de familia (media de 1,56 personas por familia) (cuadro 6).

Se comprobó que las técnicas lúdicas participatorias se adaptan a la forma de ser del campesino y permiten cambios (cuadro 7). Suscribieron compromisos para colaborar en el control de la zoonosis 992 familias $(69,7 \%)$. El cumplimiento de esos compromisos por parte de las 200 familias que integraron el grupo de casos se presenta en el cuadro 8.

En relación con los equipos de profesionales y técnicos, se capacitaron 27 técnicos de extensión agrícola, 276 profesionales de la salud, 201 auxiliares técnicos y 453 profesores rurales. Los resultados de la evaluación del proceso educativo entre estos individuos todavía no están disponibles. Al estar estas dos últimas instancias coordinadas a través de la Comisión Mixta Salud-Educación, quedaron sentadas las bases para que la red educativa sobre hidatidosis/equinococosis se pusiera en marcha en la VII Región.

El programa proporcionó una cobertura de $100 \%$ a los equipos de atención primaria rural y a 143 escuelas rurales adscritas al Programa de Mejoramiento de la Calidad y la Equidad de la Educación Rural, con un número de 4400 alumnos beneficiados cada año. Se presentaron 46 proyectos de transferencia al aula con diferentes metodologías activas para iniciar la educación sobre el tema en la VII Región y los profesores de educación básica evaluaron los conocimientos de 743 alumnos acerca de esta zoonosis. Sin embargo, los resultados de estas evaluaciones todavía no han podido ser analizados por falta de financiamiento.

\section{DISCUSIÓN}

Este trabajo utilizó un enfoque multifactorial, multisectorial y multiprofesional para abordar esta zoonosis, siguiendo las experiencias de países que han obtenido éxito en su control $(6,7)$. Partiendo de acuerdos con autoridades locales se capacitó a profesionales 
CUADRO 7. Evaluación ${ }^{a}$ de conocimientos, prácticas y hábitos relacionados con el control de la hidatidosis en los casos sometidos al proceso educativo y en los controles no sometidos a él. VII Región, Chile, 1992-1997

\begin{tabular}{|c|c|c|c|c|c|}
\hline \multirow[b]{2}{*}{ Tema } & \multicolumn{2}{|c|}{$\begin{array}{c}\text { Controles } \\
(n=95)\end{array}$} & \multicolumn{2}{|c|}{$\begin{array}{c}\text { Casos } \\
(n=200)\end{array}$} & \multirow[b]{2}{*}{$P^{\mathrm{c}}$} \\
\hline & $\begin{array}{l}\text { Puntuación } \\
\text { media }\end{array}$ & $\mathrm{DE}^{\mathrm{b}}$ & $\begin{array}{l}\text { Puntuación } \\
\text { media }\end{array}$ & DE & \\
\hline Ciclo evolutivo & 0,338 & 0,376 & 0,850 & 0,447 & $<0,001$ \\
\hline Mecanismos de transmisión & 0,475 & 0,633 & 1,562 & 0,357 & $<0,001$ \\
\hline Medidas de prevención (conocimientos) & 1,267 & 0,668 & 1,399 & 0,555 & $>0,05$ \\
\hline Medidas de prevención (prácticas) & 0,505 & 0,563 & 0,455 & 0,583 & $>0,05$ \\
\hline Medidas de control (conocimientos) & 1,168 & 0,878 & 1,476 & 0,632 & $<0,05$ \\
\hline Medidas de control (prácticas) & 0,565 & 0,571 & 1,235 & 0,835 & $<0,001$ \\
\hline
\end{tabular}

a La evaluación se hizo mediante una serie de preguntas relacionadas con cada uno de los temas enunciados.

${ }^{b} \mathrm{DE}=$ desviación estándar.

c Valor $P$ obtenido con la $t$ de Student.

CUADRO 8. Evaluación de compromisos para el cuidado personal y comunitario en el control de la hidatidosis: proporción de cumplimiento en 200 participantes en el proceso educativo. VII Región, Chile, 1992-1997

\begin{tabular}{|c|c|c|c|c|}
\hline \multirow[b]{2}{*}{ Compromiso } & \multicolumn{2}{|c|}{$\begin{array}{c}\mathrm{Se} \\
\text { comprometen }\end{array}$} & \multicolumn{2}{|c|}{ Cumplen } \\
\hline & $\mathrm{N}^{\circ}$ & $\%$ & $\mathrm{~N}^{\circ}$ & $\%$ \\
\hline Difundir el conocimiento adquirido a otros vecinos o familiares & 200 & 100 & 163 & 81,5 \\
\hline No alimentar con vísceras a los perros de la casa u otros & 165 & 82,5 & 139 & 84,2 \\
\hline Administrar el tratamiento indicado (prazicuantel) a sus perros & 161 & 80,5 & 64 & 39,8 \\
\hline Evitar que el perro salga a comer a otros lugares & 136 & 68,0 & 91 & 66,9 \\
\hline Organizarse para comprar prazicuantel en grupo & 117 & 58,5 & 8 & 6,8 \\
\hline
\end{tabular}

y técnicos agropecuarios, de salud y de educación, prestando especial atención a los aspectos del conocimiento que cada uno de estos grupos de profesionales está más capacitado para aplicar de acuerdo con sus competencias. Se trabajó en los hospitales provinciales coordinando acciones con médicos y enfermeras para la acogida y control de pacientes e implementando técnicas diagnósticas con los expertos de laboratorios clínicos. Sobre el terreno se mancomunaron esfuerzos entre médicos, veterinarios, técnicos agrícolas, técnicos paramédicos y educadores, coordinados con personal de los servicios de salud del ambiente, equipos de salud de atención primaria, especialmente postas rurales, y escuelas rurales.

El análisis general de los resultados muestra una población estudiada muy representativa del universo. La distribución porcentual por edades es similar a la de Chile y a la de la VII Región. El índice de personas por familia $(4,6)$ sobrepasa ligeramente al de la VII Región $(4,4)$ y al de Chile $(4,3)$. Según el nivel de escolaridad, la mayoría de los participantes (casi el doble que en la totalidad del país) solo habían recibido enseñanza básica, factor que se ha asociado a la hidatidosis debido a las dificultades con las que se encuentran las personas con bajo nivel de abstracción para adquirir conocimientos sobre esta (20\%) fue superior al del país (14\%) y al de la Región (16\%) al inicio del estudio (1992) (15). Los juegos utilizados en el proceso educativo fueron diseñados teniendo en cuenta esta desventaja y se elaboraron con dibujos y textos fáciles, por lo que no representaron una limitazoonosis. El índice de analfabetismo ción para la generalidad de los asistentes, situación que se ha explicado en un estudio sobre analfabetismo funcional en otra zona rural de Chile (14).

El estudio diagnóstico de la hidatidosis humana permitió establecer la tasa de prevalencia para la población asintomática, presuntamente sana, de una zona endémica: la VII Región. De acuerdo con los resultados de las pruebas serológicas (HAI y ELISA), los 42 individuos con ambas pruebas positivas representan una seroprevalencia de 754,6 por 100000 habitantes. En 16 de los 26 casos $(61,5 \%)$ con HAI y ELISA positivas que se sometieron a la realización de radiografías de tórax y ecografías abdominales, los resultados de estas pruebas de visualización fueron compatibles con el diagnóstico de hidatidosis hepática (10 casos) o pulmonar (6 casos), cifra que es superior al 50,9\% comunicado por otros autores (16).

A pesar de que en nuestro estudio se utilizaron las pruebas de ELISA y HAI, con una especificidad de 95,5\% (9) y $96,6 \%$ (8), respectivamente, las pruebas de visualización resultaron negativas en $38,5 \%$ de los casos con ambas pruebas serológicas positivas. Esta discrepancia entre los resultados de las pruebas serológicas y de visualización podría deberse a la presencia de anticuerpos inespecíficos que aumentarían la incidencia de seropositividad, a la existencia de quistes muy pequeños no detectables por radiografía o ecografía, a localizaciones extraabdominales y extratorácicas o, excepcionalmente, a la curación espontánea del quiste (16).

Los resultados de la prueba de "dot" ELISA no fueron considerados como criterio diagnóstico por su inespecificidad. Aunque la DD5 posee una alta especificidad, el escaso número de casos sometidos a ella en este estudio impidió su utilización como criterio diagnóstico.

Los 26 pacientes con imágenes positivas fueron referidos al hospital para ser intervenidos quirúrgicamente, pero 7 no acudieron a la cita; los otros 9 casos fueron confirmados quirúrgicamente, lo cual representa una prevalencia de 161,7 por 100000 habitantes (9/5 566), con un promedio anual para el período de estudio de 32,3 por 100000 , cifra que 
es casi 8 veces mayor que las cifras oficiales de la VII Región y 14 veces mayor que las de Chile, que al inicio del estudio eran de 4,3 y 2,3 por 100000 , respectivamente (2). Aplicando esta tasa a la población inicial del estudio con residencia rural (336 173 habitantes) existiría la probabilidad de localizar 108 personas portadoras de quistes hidatídicos. De poder contar con un programa nacional de control de esta infección y con tratamiento médico, muchos casos podrían ser identificados y tratados tempranamente, con lo cual se evitaría la intervención quirúrgica de quistes complicados, que tanta letalidad provoca (17).

El diagnóstico del huésped definitivo se realizó mediante la administración oral de bromhidrato de arecolina, procedimiento que, si bien es lento, engorroso y pone en riesgo de infección al personal que lo realiza (12), permitió obtener reacciones positivas en $81,9 \%$ de los perros estudiados, rendimiento que se puede considerar bueno (12). La tasa media de prevalencia de infección por E. granulosus fue de $11 \%$, cercana a la registrada en Uruguay en las regiones de Durazno (19,7\% en 1955) (18) y Tacuarembó (19\% en 1992) (19), pero muy inferior a la de otras Regiones chilenas, como la XI (54,4\% en 1980) (3) y la XII $(70,9 \%$ en 1978) (20), o a la registrada en otros países, como China $(71 \%)(21,22)$. La cifra fue más elevada en algunas localidades de mayor marginalidad, mayor ruralidad o peores condiciones epidemiológicas. Así, en Cauquenes se detectó una tasa media de infección de 17,7\%: 24,2\% en las grandes propiedades agrícolas y 15,9\% en parcelas. En las provincias de Linares, Talca y Curicó se registró una tasa media inferior a la de Cauquenes, aunque en estas provincias existen localidades con un alto porcentaje de infección canina, como Retiro (28,9\%), Villa Alegre $(16,7 \%)$ o Empedrado $(15,6 \%)$. Esta situación de alta prevalencia resulta preocupante por el riesgo que representa para la salud de la población, y también por las elevadas pérdidas que origina en la productividad animal. En cinco años (1990-1995), se detectó hidatidosis en 13,0\% de los bovinos, $4,4 \%$ de los ovinos y $4,2 \%$ de los porcinos sacrificados en los mataderos de la VII Región (cuadro 3). Estas cifras son importantes por su magnitud, pero, además, se debe considerar que la inmensa mayoría de los animales sacrificados provienen de los campos y criaderos de la zona, hecho que confirma la alta prevalencia de la enfermedad en el ganado de la VII Región. A estas cifras oficiales habría que agregar los casos detectados mediante encuestas epidemiológicas de población rural, donde se determinó que es una costumbre muy arraigada en la zona la matanza casera, tanto para consumo propio como con fines comerciales. De acuerdo con los datos proporcionados por las entrevistas a los cabezas de familia, en los hogares campesinos se sacrifican cerca de 93000 ovinos y 53000 cerdos por año. Como todos los animales para consumo propio son criados cerca de las casas y, por lo general, sus vísceras son consumidas por los perros, se mantiene así un ciclo doméstico que permite una alta endemia de esta zoonosis en la VII Región.

Si solo se consideran las pérdidas económicas basadas en las cifras oficiales obtenidas por decomisos en mataderos, cada año se pierden en la VII Región cerca de 10000 kilos de hígado de vacuno, que se vende en el mercado nacional a cerca de mil pesos chilenos (Ch\$) el kilo. Por tanto, se estarían perdiendo anualmente cerca de $\mathrm{Ch} \$ 10 \mathrm{mi}$ llones por decomiso de estas vísceras. Las pérdidas por el desperdicio de vísceras en la matanza domiciliaria alcanza los Ch\$ 9,3 millones anuales. De este modo, al año se pierden en la región cerca de Ch\$19 millones por decomiso y/o eliminación de las vísceras infectadas. Si a esto se le agrega la menor productividad de los animales infectados, cercana a 5\% y que representa unos Ch\$ 100 millones de pérdidas anuales, las pérdidas totales en la VII Región alcanzarían los Ch\$ 119 millones anuales, es decir, alrededor de US\$ 265000 (US\$ $1=\mathrm{Ch} \$ 450$ ).

Aunque se ha comprobado que la medida de control más efectiva para reducir la prevalencia de la equinococosis es el tratamiento del perro, la medida preventiva fundamental es la educación, a fin de que las personas hagan suyo el compromiso de cambiar hábitos y conductas de riesgo (23). En la mayoría de los países donde se han iniciado programas de control se ha puesto énfasis en las campañas de divulgación educativa; en otros se han hecho valoraciones de las posibilidades y limitaciones de la educación sanitaria para proponer alguna metodología educativa $(18,24-26)$ y se han probado materiales educativos de carácter lúdico, preferentemente en niños, y folletos en adultos $(22,24,25)$. Sin embargo, no se han descrito experiencias con metodologías participatorias.

En lo que respecta a la evaluación de los conocimientos de la población, los resultados obtenidos en cuanto a prácticas de crianza de perros, y especialmente en lo que se refiere a su alimentación con vísceras infectadas, coinciden con lo señalado en informes tanto extranjeros como nacionales. Los cabezas de familia que declararon no saber nada (55\%) o que tenían conceptos errados sobre la equinococosis/ hidatidosis $(6,4 \%)$ representaron $61,4 \%$ de nuestra población, cifra comparable a la obtenida a nivel nacional en Chile $(67,3 \%)$ (27), algo más elevada que la encontrada en Uruguay $(56,6 \%)$ (19) y más baja que la registrada en China $(75 \%)$ (21). Cabe destacar que Uruguay cuenta con programas establecidos a nivel nacional, mientras que las experiencias de nuestro grupo y de China se refieren a programas regionales y muestrales. En este último país, la evaluación de la intervención educativa fue positiva, al igual que en nuestro estudio. Tomando en consideración el bajo nivel de escolaridad de la muestra con la que se trabajó y el hecho de que solo se realizó una intervención educativa, a diferencia de China, donde hubo más de tres (22), se considera que los resultados son prometedores. No obstante, no se pudo efectuar vigilancia en relación con las prácticas de riesgo por tener esta investigación solo cinco años para su desarrollo. Se espera que, en el futuro, los equipos locales puedan comunicar resultados al respecto. Quedan por evaluar los efectos del olvido, que no deberían ser tan marcados si las familias educadas y los equipos capacita- 
dos siguen reforzando lo realizado por el equipo del estudio.

La cobertura alcanzada en la capacitación profesional ha sido similar a la experiencia chilena de la XII Región, donde el programa de control de la hidatidosis se implantó hace más tiempo (20) y, especialmente en lo que respecta a profesores rurales, se espera tener una proyección en las generaciones jóvenes durante algunos años.

Una experiencia inédita es la de establecer compromisos con las familias educadas. Fue interesante comprobar que $70 \%$ de los campesinos educados se sintieron en libertad de decidir sus compromisos para colaborar en controlar y prevenir la zoonosis. Lo observado es que muchos se comprometieron más allá de lo que en la realidad pudieron cumplir. No obstante, la gran mayoría cumplió con difundir el conocimiento aprendido, lo que favorece la multiplicación de esfuerzos, transformándose en las fuentes primarias de conocimientos para otras familias (27). Si cada una de las 992 familias que suscribieron compromisos traspasara sus conocimientos a un miembro de otras tantas familias por una sola vez, a los 2427 individuos educados en primera instancia debe- ríamos agregar otros 992, lo cual nos daría un total de 3419 personas informadas sobre el tema.

Este estudio permitió establecer nexos iniciales entre los servicios de salud, educación y agropecuarios que deben ser mantenidos para afianzar los logros obtenidos. Para este fin se debe contar con un financiamiento permanente que permita evaluar periódicamente el impacto de las acciones iniciadas. Sobre la base de los resultados de esta investigación, sería importante considerar la vigilancia activa de la hidatidosis y poder concentrar los esfuerzos en un plan nacional.

\section{REFERENCIAS}

1. Serra I, Reyes H. Hidatidosis humana en cuatro países de Sudamérica. Bol Oficina Sanit Panam 1989,106:525-530.

2. Chile, Ministerio de Salud. Anuarios de Notificación Obligatoria (1961-1994). Santiago: Ministerio de Salud; 1962-1995.

3. Chile, Ministerio de Agricultura, Servicio Agrícola y Ganadero. Proyecto de control de hidatidosis en la XI Región. Santiago: Ministerio de Agricultura; 1982

4. Campano S. Control de la hidatidosis/ equinococosis en la XII Región de Chile. Noticias Médico-Veterinarias 1986;2:135-144.

5. Apt W, Pérez C, Rycke PH. Tratamiento no convencional de la hidatidosis humana. Rev Med Chil 1996;124:1385-1389.

6. Gemmel MA, Lawson JR. Epidemiology and control of hydatid disease. En: Thompson R, ed. The biology of Echinococcus and hydatid disease. Boston: George Allen \& Unwein Publisher; 1986. pp. 189-216.

7. Gemmel MA, Lawson JR, Roberts MC. Toward global control of cystic and alveolar hydatid diseases. Parasitol Today 1987;3: 144-151.

8. Knierim F, Saavedra P. Técnicas de la reacción de hemaglutinación aplicada al diagnóstico serológico de las parasitosis. Bol Chil Parasitol 1966;21:39-44.

9. Vargas D, Sabbe G, Castro R, Pérez C, Apt W, De Rycke PH. Implementation of an ELISAtest for the diagnosis of human hydatid disease. Rev Parasitol 1995;55:223-226.

10. Coltorti EA, Varela-Díaz VM. Detection of antibodies against Echinococcus granulosus arc 5 antigens by double diffusion test. Trans Roy Soc Trop Med Hyg 1978;72:226-229.

11. Vargas D, Pérez C, Gutiérrez F, Parada M, Cortés P, Retamal C, et al. Dot ELISA en el diagnóstico presuntivo de la hidatidosis humana. Parasitol al Día 1996;20:16-19.

12. Schantz P. Guía para el empleo del bromhidrato de arecolina en el diagnóstico de la infección por Echinococcus granulosus en el perro. Bol Chil Parasitol 1973,28:81-90.

13. Chile, Instituto Nacional de Estadísticas. Resultados generales. Censo de Población y Vivienda, Chile, 1992. Santiago: Instituto Nacional de Estadísticas; 1992. pp. 328-335.

14. Galdamez O, Cortés P, Vargas D, Rodríguez J, Vega F, Pérez C, et al. Variables epidemiológicas asociadas a hidatidosis en población rural asintomática. Parasitol al Día 1997;21:7-13

15. Novoa T. Analfabetismo funcional. Rev Educ Chile 1992;202:35-38.

16. Coltorti E, Fernández E, Marguet E, Siozzina J, Guarnera E. Detección de portadores asintomáticos de quistes hidatídicos: aumento de la especificidad del ensayo inmunoenzimático. Rev Inst Med Trop São Paulo 1990; 34:275-284.

17. Sapunar J, Rappaport J, Sapunar J, Cunsille F. Quiste hidatídico hepático. Características clínicas, factores pronósticos y resultados quirúrgicos. Parasitol al Día 1989;13: 53-63.

18. Dibarboure L, Botta B, Paolillo E, Cohen $\mathrm{H}$ Rodríguez $\mathrm{O}$, Bibbo L et al. Hidatidosis: caracterización de dos poblaciones del Departamento de Durazno. Libro de Resúmenes del X Congreso Latinoamericano de Parasitología. I Congreso Uruguayo de Parasitología. Montevideo, Uruguay, 1991. pp. 357.

19. Asociación Internacional de Hidatidología. Noticias de la hidatidosis. El programa de Tacuarembó (Uruguay) de control de la hidatidosis. Asociación Internacional de Hidatidología 1996;14:13-17.

20. Chile, Ministerio de Agricultura, Servicio Agrícola y Ganadero: evaluación del Proyecto Control de la Hidatidosis en la XII Región de Chile, 1983-1987. Santiago: Ministerio de Agricultura; 1989

21. Chi P, Zhang W, Zhang Z, Hasyer Z, Liu F, Ding Z, et al. Cystic echinococcosis in the Xin-
jiang/Uygur Autonomous Region, People's Republic of China. I. Demographic and epidemiologic data. Trop Med Parasitol 1990; 41:157-162.

22. Andersen LF, Tolley HD, Shantz P, Chi P, Liu F, Ding Z. Cystic echinococcosis in the Xinjiang/Uygur Autonomous Region. People's Republic of China. II. Comparison of three levels of a local preventive and control program. Trop Med Parasitol 1991;42:1-10.

23. Neghme RA. Impacto de las enfermedades parasitarias y rol de la educación en salud. Santiago: Instituto de Chile, Academia de Medicina; 1998.

24. Omstein J, Cabrera P. Bases metodológicas para el estudio interdisciplinario de la hidatidosis. Libro de Resúmenes del X Congreso Latinoamericano de Parasitología. I Congreso Uruguayo de Parasitología. Montevideo, Uruguay, 1991. pp. 353.

25. Thomas MV. Actividades de Educación Sanitaria. I Seminario Internacional de Hidatidosis, Punta Arenas, Chile, 1998.

26. Bibbó L, Frau A, Botta B, Colina R, Cornejo G, Ramos M. Concepciones de la población sobre hidatidosis y su vinculación con la educación. Libro de Resúmenes del X Congreso Latinoamericano de Parasitología. I Congreso Uruguayo de Parasitología. Montevideo, Uruguay, 1991. pp. 359.

27. Ernst $S$, Thiele K. Conocimiento específico sobre hidatidosis en la población de un sector rural de la Provincia de Valdivia, Chile. Bol Chil Parasitol 1985,40:42-44.

Manuscrito recibido el 12 de octubre de 1998 y aceptado para publicación, tras revisión, el 29 de junio de 1999. 
ABSTRACT This study was designed to embrace three areas: a) serologic and radiologic diagnosis and surgical treatment of hydatidosis in an asymptomatic human population, b) animal diagnosis and the treatment of dogs, and c) evaluation of extent of knowledge and performance of educational interventions among rural families and health, livestock, and education professionals and technicians, in order to help control the disease transmission cycle. Indirect hemagglutination and ELISA tests were performed on 5556 apparently healthy people. Of these, $42(0.8 \%)$ had positive results on both tests, for a seroprevalence of 754.6 per 100 000. These 42 subjects were scheduled for liver ultrasonography and a chest x-ray; of the 26 who complied, 16 showed images compatible with a hydatid cyst. Those 16 cases were sent to the hospital for surgery. In 9 of the cases the diagnosis was confirmed surgically, for a prevalence of 161.7 per 100 000. Arecoline hydrobromide was administered as a laxative to 2358 dogs to detect the strobilar form of Echinococcus granulosus, and positive results were found in $11 \%$ of the dogs. Official data for slaughterhouses indicated the presence of hydatid cysts in $13 \%$ of the cattle, $4.4 \%$ of the sheep, and $4.2 \%$ of the pigs slaughtered in the region. The educational program included an evaluation of the extent of knowledge by surveying heads of household; an educational intervention among families through an informal active participatory process using educational games, in which 1082 families participated; and an educational intervention with professionals and technicians using distance and in-person approaches. To evaluate the program, the results of knowledge tests before and after educational interventions with 200 families (cases) were compared with those from 95 families who did not participate (controls). Of the 1423 heads of household initially surveyed about their knowledge of echinococcosis/hydatidosis, 783 of them (55\%) said they knew nothing about the infection. It was found that the participatory educational games were well adapted to the lifestyle of people from rural areas and made change possible. Training was provided to 276 health professionals, 201 technical assistants, and 453 rural teachers. The program reached $100 \%$ of the staff members of the area's rural primary health care services. 